ISSN: 2215-2644

revedu@gmail.com

Universidad de Costa Rica

Costa Rica

\title{
Análisis de los procesos adaptativos en estudiantes migrantes de postgrado de una Universidad pública del sur de Chile
}

Guevara Ortiz, Odaly Ivette; Alfaro Urrutia, Jorge Eduardo

Análisis de los procesos adaptativos en estudiantes migrantes de postgrado de una Universidad pública del sur de Chile

Revista Educación, vol. 45, núm. 2, 2021

Universidad de Costa Rica, Costa Rica

Disponible en: https://www.redalyc.org/articulo.oa?id=44066178016

DOI: https://doi.org/10.15517/revedu.v45i1.43617

\section{(c) (1) $\Theta(9$}

Esta obra está bajo una Licencia Creative Commons Atribución-NoComercial-SinDerivar 3.0 Internacional. 


\section{Análisis de los procesos adaptativos en estudiantes migrantes de postgrado de una Universidad pública del sur de Chile}

Probing into the Adaptation Process of Postgraduate Migrant Students at a State University in Southern Chile

Odaly Ivette Guevara Ortiz

Universidad Pedagógica Nacional Francisco Morazán de

Honduras, Honduras

odaly1385.g@gmail.com

iD https://orcid.org/0000-0002-9491-449X

Jorge Eduardo Alfaro Urrutia

Departamento de Administración de Educación Municipal,

Chile

jorgeeduardoau@gmail.com

(D) https://orcid.org/0000-0003-3711-6536
DOI: https://doi.org/10.15517/revedu.v45i1.43617

Redalyc: https://www.redalyc.org/articulo.oa? $\mathrm{id}=44066178016$

Recepción: 13 Septiembre 2020

Aprobación: 22 Octubre 2020

\section{Resumen:}

Chile es uno de los países de Latinoamérica que más ha visto incrementado su flujo de estudiantes provenientes de otros países (Vásquez-De Kartzow, Castillo-Duran y Lera, 2015). La reciente implementación de una política para estudiantes migrantes aprobada por el Ministerio de Educación Chileno (MINEDUC, 2018) da cuenta de un terreno incipiente en el cual resulta interesante analizar los procesos de adaptación académica de estudiantes extranjeros/as de postgrado de una universidad pública del sur de Chile. Para ello se llevó a cabo un estudio de caso cualitativo en el cual se entrevistó a 7 estudiantes extranjeros y a 3 de sus docentes de postgrado. Los resultados dan cuenta de múltiples factores pedagógicos y externos, los cuales, permeados por la cultura, influyen positiva o negativamente en el proceso de aprendizaje. Se concluye que, a pesar de las similitudes idiomáticas, los y las estudiantes migrantes enfrentan una serie de barreras relacionadas con las variantes lingüísticas y relacionales que pueden hacerlos/las vivir procesos de asimilación o aislamiento cultural. El fortalecimiento de las prácticas institucionales es la principal recomendación para una mayor inclusión.

Palabras ClaVE: Estudiantes Migrantes, Adaptación, Estrategias de enseñanza, Migración en Chile.

\section{Abstract:}

Chile is one of the Latin American countries that has experienced a significant rise of migrant students in its student body (Vásquez-De Kartzow, Castillo-Duran and Lera, 2015). The recent implementation of a migrant student policy approved by the Chilean Ministry of Education (MINEDUC, 2018) reveals an incipient terrain in which it is interesting to analyze the academic adaptation processes of foreign postgraduate students from a state university in the South of Chile. A qualitative case study was executed whereby seven foreign students and three of their postgraduate lecturers were interviewed. The results reveal pedagogical and external factors which, imbedded by culture, have a positive or negative influence on learning. Yet, despite idiomatic similarities, migrant students face many linguistic and relational barriers through which they may experience assimilation or cultural isolation. Strengthening institutional practices is the main recommendation for greater inclusion.

KEYWORDS: Migrant Students, Adaptation, Teaching Strategies, Chilean Migration.

\section{INTRODUCCIÓN}

La migración es entendida por la Organización Internacional para las Migraciones como "el movimiento de población hacia el territorio de otro estado o dentro del mismo, que abarca todo movimiento de personas sea cual fuere su tamaño, su composición o sus causas" (OIM, 2018 p.38). La misma organización ha cifrado la 
cantidad de personas migrantes en 272 millones (OIM, 2019),y si bien la mayor parte lo hace de forma segura y ordenada, de acuerdo con la Organización de las Naciones Unidas, existe un porcentaje que lo hace en un contexto de vulnerabilidad (ONU, 2017) y el cual enfrenta numerosas situaciones de violencia (Torres, 2012).

En América del Sur, la Organización para la Cooperación y el Desarrollo Económico ha situado a Chile como uno de los tres principales países de destino migratorio de la región (OCDE, 2017), principalmente por contar con un sólido marco macroeconómico cuyo crecimiento, de acuerdo con el Banco Mundial (2017), fue de 1,7\%. De acuerdo con el Instituto Nacional de Estadísticas, Chile ha alcanzado una población de migrantes superior al millón de personas (INE, 2019). En ese sentido, naciones con grandes libertades económicas atraen a personas migrantes con más altos niveles educativos (Mulholland y Hernández-Julian, 2013). En el caso del estudiantado que migra, las situaciones vividas son variadas, pero encuentran un punto en común en términos del apoyo social (Nada y Araujo, 2018).

En Chile, ante la presencia de una nueva población estudiantil migrante, surge la necesidad de revisar los sistemas educativos en función de esta nueva población creciente. Los estudios más recientes sobre educación y migración tienden a concentrarse en educación primaria describiendo fortalezas, obstaculizadores y propuestas (Joiko y Vásquez, 2016; Mondaca, Muñoz, Gajardo, Gairín, 2018; Poblete, 2018; Aninat y Vergara, 2019), sin embargo, es escasa la información sobre la experiencia en educación superior, especialmente en el nivel de postgrado.

Por tal motivo se ha llevado a cabo este estudio, el cual problematiza respecto de los procesos adaptativos que vive el estudiantado migrante de postgrado, y cómo estos al interactuar con las condiciones del país de destino favorecen u obstaculizan el aprendizaje.

Se presentan a continuación antecedentes respecto del fenómeno de la migración y su efecto en el aprendizaje. Asimismo, se esclarecerá cómo esa interacción entre el proceso de adaptación del estudiante y su relación con el aprendizaje constituye un fenómeno poco abordado por la literatura actual. Posteriormente, se dará cuenta de los aspectos metodológicos para luego presentar los resultados y discusiones del estudio en cuestión.

Se espera con esta investigación aportar lineamientos para construir prácticas pedagógicas y políticas institucionales más inclusivas desde la voz de quienes viven dichos procesos.

\section{Migración en Chile}

Como se mencionó anteriormente, existe un grupo importante de personas que migra por razones sociales en situación de gran vulnerabilidad; sin embargo, también hay un grupo que migra por motivos económicos y educativos. En ese sentido, la OIM (2018) ha establecido que la necesidad de formarse en otro país aparece como una motivación para la migración, lo cual se ve reflejado en una movilidad de 4.8 millones entre países a nivel global.

Los principales países en que se da esta movilidad estudiantil son Estados Unidos, los pertenecientes a la Unión Europea y, en el caso de Latinoamérica, Brasil, Chile y Argentina son los principales destinos (Organización de las Naciones Unidas para la Educación, Ciencia y la Cultura [UNESCO], 2019).

Para Chile, este fenómeno resulta reciente y creciente, lo cual queda reflejado en el ingreso de 700 mil personas, aumentando desde un 2,5\% a un 5,9\% en tan solo tres años (Aldunate, Contreras, de la Huerta y Tapia, 2018). Asimismo, en Chile, el Instituto Nacional de Estadística [INE] (2019) describe el aumento exponencial de la migración en el país, siendo una persona migrante por cada mil en 2005, entre 1 y 2 por cada mil en 2014; 3,10 por cada mil para 2015; 6,9 por cada mil en 2016 y 11,6 por cada mil en 2017.

Basados en la Declaración de los Derechos Humanos (Organización de las Naciones Unidas [ONU], 1948), en la Declaración de New York (ONU, 2016) y los Objetivos del Desarrollo Sostenible 2030 (Comisión Económica para América Latina [CEPAL], 2018), Chile ha ido adecuando sus normativas, 
visibilizadas en la Constitución de la República (Ley N²0.050, 1980), Ley General de Educación (Ley N - 20.370, 2009), y concretándose en la promulgación de la Política Nacional de Estudiantes Extranjeros (MINEDUC, 2018). Ello da cuenta de voluntades legislativas para responder a las necesidades educativas que subyacen en la población migrante.

Sin embargo, estas voluntades contrastan con los resultados de la Encuesta Internacional sobre Enseñanza y Aprendizaje en la Escuela, donde se observó que solo el 16\% de los docentes de primer ciclo habían emprendido actividades de formación multicultural o bilingüe (UNESCO, 2018). De la misma forma, la OCDE (2017) realizó sugerencias a Chile entre las cuales destaca la necesidad de contar con estrategias para enseñar en un entorno multicultural y multilingüe, considerando esta una de las áreas en que los docentes tienen importantes necesidades de desarrollo profesional.

Respecto de la Educación Superior, se ha evidenciado un aumento de un 13\% de estudiantes migrantes para el año 2019 (Consejo Nacional de Educación [CNED], 2019), representando una cifra de 16000 estudiantes extranjeros matriculados en diferentes instituciones de educación superior de Chile. Esto en un contexto de una educación superior chilena marcada por enfoques económicos que se está adaptando desde un modelo de autofinanciamiento a un modelo de gratuidad, el cual abre desafíos vinculados con la educación de personas de sistemas socioeconómicos más bajos, quienes anteriormente no tenían acceso a dicho sistema (Espinoza y González, 2017).

\section{Adaptación}

$\mathrm{Al}$ igual que cualquier persona que enfrenta un cambio importante, las personas que migran inician un proceso de adaptación en función de lo ya conocido o de la situación pasada respecto de la situación presente o futura. En ese sentido, la adaptación, desde la psicología social, se ha definido como una función recíproca entre el individuo y su medio, caracterizada por cómo la conducta del individuo se orienta hacia el bienestar y el equilibrio entre las necesidades y las circunstancias (Kulshrestha, 1979; Jiménez y López-Zafra, 2011; Builes, Manrique y Henao, 2017).

Cuando una persona migra desde su país hacia un nuevo destino, existe una necesidad de equilibrar múltiples necesidades (económicas, afectivas, sociales), independientemente sean los motivos por los cuales se haya migrado, para alcanzar un bienestar presente y/o futuro. En ese sentido, y abocado al tema educativo, la adaptación resulta un factor necesario para el éxito académico, puesto que se ven influidas variables como las redes de amistad, la integración y la autoestima (Castillo, Santa Cruz y Vega, 2018). La adaptación puede ser psicológica cuando se relaciona con la salud mental de la persona o sociocultural cuando se relaciona con la gestión de la propia vida en el contexto intercultural (Arenas y Urzúa, 2016).

El elemento social es el primer factor en verse afectado al momento de realizar un proceso migratorio en contexto educativo (Castro y Lupano, 2013; Salazar-Salas, 2015). Esto se materializa en la necesidad de construir redes de apoyo social en el país de acogida, enfrentar ciertos niveles de discriminación y estereotipos, así como la regulación del estrés y ansiedad. Ferrer, Palacios, Hoyos y Madariaga (2014) describen procesos de aculturación que son parte de la adaptación de personas migrantes, como son los de contenido (sentimientos, actitudes y conductas), de proceso (relaciones) e interactivos, que relacionan los dos primeros.

De esta forma, Williamson (2019) establece la necesidad de promover en contextos con estudiantes migrantes, ajustes y estrategias de adaptación, las cuales son facilitadas por los cuerpos docentes y desarrolladas por los y las estudiantes. Así, las estrategias de adaptación académica se entienden como mecanismos que los y las estudiantes, y/o las instituciones utilizan conscientemente para alcanzar determinados objetivos (Bustos, 2016).

De acuerdo con el estudio de Aninat y Vergara (2019), los y las estudiantes migrantes se concentran en establecimientos determinados, mientras que las y los recién llegados tienen resultados similares o levemente inferiores a sus pares, pero quienes llevan más tiempo obtienen resultados en promedio superiores. Tienen 
mejores resultados en matemáticas que en lenguaje. El mismo estudio describe que la concentración de estudiantes migrantes ha favorecido la convivencia escolar, sin embargo y a pesar de su buen desempeño, el acceso a la educación superior es desigual.

En ese sentido, Stefoni y Corvalán (2019) señalan que la mayoría de las investigaciones sobre migración estudiantil en Chile son realizadas en términos sociológicos o antropológicos, pero hace falta profundizar respecto de los procesos pedagógicos y de aprendizaje que viven quienes migran y estudian.

Considerando el creciente y exponencial flujo de estudiantes migrantes hacia Chile (Instituto Nacional de Estadísticas [INE], 2019), sumado a la falta de literatura respecto de estos procesos en educación de postgrado es que se propone una investigación que tiene por finalidad analizar los procesos de adaptación académica de estudiantes extranjeros/as de postgrado.

\section{Procedimientos metodológicos}

Se desarrolló una investigación bajo un paradigma interpretativo y un enfoque cualitativo (Banks, 2010; Niño, 2011), puesto que se pretende describir e interpretar la realidad percibida por un grupo de personas desde sus propias vivencias y experiencias. A través de un Estudio de Caso (Mackernan, 1989) y de una población de 10 estudiantes, participaron de manera intencionada (Ruíz, 2007) 7 estudiantes extranjeros/ as (4 varones y 3 mujeres) de postgrado en diferentes disciplinas y provenientes de países de Latinoamérica (Venezuela, México, Colombia) y África (Mozambique) y de un promedio de edad de 28 años, además de 3 docentes chilenos/as (dos varones y una mujer), quienes les realizaron clases en el postgrado en áreas de ciencias sociales, educación y matemáticas. La universidad seleccionada es la única universidad pública de la región y que, asimismo, tiene la mejor puntuación, especialmente en su línea de postgrado.

La información se recogió a través de una entrevista semiestructurada individual (Escobar y Cuervo, 2008), la cual fue diseñada considerando experiencias recopiladas de los antecedentes teóricos revisados y con énfasis en la experiencia del grupo de estudiantes. Previo a la entrevista, los y las participantes firmaron un consentimiento que garantizó anonimato y confidencialidad. El protocolo de entrevista fue sometido a juicio de experto.

Las entrevistas, realizadas en dependencias de la universidad por la investigadora principal, fueron grabadas en audio y posteriormente transcritas para luego ser sometidas a un análisis de contenido (Monje, 2011; Tinto 2012), el cual permite establecer relaciones desde la semántica y significados en los enunciados.

De esta forma, el análisis de contenido se realizó identificando acciones, sentimientos o procesos que daban cuenta de procesos de adaptación tanto desde la persona como desde el contexto, asimismo, se identificaron las conductas que resultaron de dichos elementos y que constituyen la adaptación en concreto. Así, desde un razonamiento inductivo, se organizaron categorías y subcategorías las cuales se presentan a continuación.

\section{Resultados}

A partir de los análisis, los resultados se organizaron en dos categorías: Procesos adaptativos del estudiantado y Estrategias para favorecerlos.

Respecto de la categoría Procesos Adaptativos, los discursos de las y los participantes se organizaron en las subcategorías Adaptación académica, Adaptación sociocultural y Adaptación psicológica (ver Tabla 1). 
TABLA 1

Procesos de Adaptación en estudiantes migrantes

$\begin{array}{cll}\text { Adaptación Académica } & \begin{array}{l}\text { Variantes lingüisticas } \\ \text { Relación con las/los docentes }\end{array} \\ \text { Procesos de Adaptación Adaptación Sociocultural } & \begin{array}{l}\text { Vivencias de discriminación } \\ \text { Adeptación Psicológica }\end{array} & \begin{array}{l}\text { Integración } \\ \text { Autorregulación } \\ \text { Aislamiento }\end{array}\end{array}$

Fuente: Elaboración propia

Respecto a la subcategoría adaptación académica, esta reúne un conjunto de situaciones que vivencian al momento de desenvolverse en la sala de clases y/o en las actividades lectivas propuestas para sus cursos. En este sentido cobran especial relevancia las diferencias idiomáticas, entre las cuales identifican el uso de modismos por parte de las y los académicos durante las clases, la velocidad del habla y variantes lingüísticas, esto queda en evidencia al señalarse que "solo que habla, modismos, habla muy rápido y usa palabras formales que debes buscar en el diccionario" (Estudiante 1, comunicación personal, 15 de enero de 2020) y "la forma de enseñar es totalmente diferente, los [profesores] de afuera que estudiaron afuera hablan despacio y no usan modismos porque entienden que los extranjeros no entienden nada para que otra persona pueda entender lo que enseña" (Estudiante 3, comunicación personal, 12 de enero de 2020). En ese sentido, se realiza una diferencia entre el y/o la profesora que tiene experiencia relacionándose con personas extranjeras y quienes no, lo cual se materializa en su forma de hablar y comunicar.

De lo anterior se ilustran procesos de adaptación académica que tienen relación con las variantes lingüísticas, es decir, con cómo el idioma y sus características culturales como la velocidad, modismos y forma de pronunciación pueden constituir un elemento que favorezca u obstaculice la adaptación académica.

En la misma línea, las y los estudiantes ven en la figura del o de la profesora a una persona que pasa a formar parte de la red de apoyo local. Esta relación, parece ser descrita de manera natural puesto que inicialmente no hay otras personas con quienes establecer el vínculo.

Yo creo que en general las personas buscamos, validación de alguien y al ser estudiante, el estudiante migrante pues un referente o su referente directo es su profesor a sus profesores, entonces al estar fuera de su contexto el estudiante carece de esta figura que le dan validación, no, la validación hacia arriba es casi exclusiva de tus profesores, no hay nadie más arriba que tú en el contexto. (Estudiante 6, comunicación personal, 8 de enero de 2020).

Así, aparece un proceso de adaptación académica que se fundamenta en la relación con las y los docentes. La posibilidad de identificarse con el o la docente permitiría una mejor adaptación.

Respecto a la subcategoría Adaptación sociocultural, los y las estudiantes referencian procesos de rechazo, "porque hasta a mi me rechazaron de habitaciones por ser negra, por tener color negra, hable con la doña en el teléfono todo bien y cuando me conoció, no te puedo, ¿Por qué? Porque no puedo, por mi color" (Estudiante 1, comunicación personal, 15 de enero de 2020) y discriminación, en tanto:

...los estudiantes que son de raza negra van a tener un problema de discriminación grave en este país, grave en provincia, probablemente en Santiago no si son iguales somos iguales yo creo que ahí hay una discriminación racial muy marcada en Chile. (Estudiante 4, comunicación personal, 12 de diciembre de 2019).

Esto se explicita principalmente en la vida social de las y los estudiantes dentro del país.

Los procesos de rechazo tienen relación con características socioculturales del entorno que recibe al estudiante y a los cuales este debe adaptarse. En este caso la experiencia es interpretada como un rechazo, el cual debe enfrentarse.

Respecto de la Adaptación psicológica, se referencian tres procesos, uno de integración, otro de autorregulación y otro de aislamiento. 
En cuanto a la integración, "Dentro del doctorado siento que [mi experiencia] ha sido positiva, porque favorablemente cada uno de mis compañeros tiene una formación distinta y la integración que hemos tenido ha sido muy buena porque siento que nos apoyamos unos a otros" (Estudiante 3, comunicación personal, $12 \mathrm{de}$ enero de 2020). La adaptación desde la integración queda caracterizada como una participación en el plano académico donde prima la relación vinculada a los estudios. En ese sentido el o la estudiante participa gracias a la relación académica que se pone a disposición por parte de los docentes.

En cuanto a la autorregulación se plantea que:

...los chilenos son muy complejos, piensan que uno les quita sus competencias, que uno le quita su inteligencia, entonces al inicio busque hablar, pero cuando uno ve la mirada de otras como que no les gusta entonces no sé, yo no entiendo a mí no me hace mal, yo no hablo, pero aprendo harto. (Estudiante 1, comunicación personal 15 de enero de 2020).

Con la finalidad de disminuir la hostilidad que percibe por parte de las relaciones establecidas con estudiantes pertenecientes a la cultura mayoritaria, el/la estudiante recurriría a procesos de adaptación psicológica de autorregulación, disminuyendo voluntariamente su participación para evitar así la relación dolorosa.

En cuanto al aislamiento, "cuando les estaba hablando de mis viajes y ya simplemente nadie más habla ya contigo, ya no son más cercanos" (Estudiante 7, comunicación personal, 10 de diciembre de 2019). En ella, la experiencia es tan negativa que el o la estudiante encuentra como estrategia de adaptación el alejarse del grupo, o bien acepta el alejamiento por parte del grupo mayoritario. El aislamiento aparecería como una respuesta de adaptación voluntaria o motivada desde lo externo, para poder mantener los estudios sin tener que ocuparse de una relación que ya ha fracasado.

Respecto a la segunda categoría, Estrategias que se utilizan para enfrentar el proceso de adaptación de los estudiantes migrantes, surgieron dos subcategorías: Estrategias docentes y Estrategias institucionales (ver Tabla 2).

TABLA 2

Estrategias para la adaptación de estudiantes migrantes

\begin{tabular}{lll} 
& & Lenguaje Neutro \\
Estrategias para la adaptación Estrategias docentes & $\begin{array}{l}\text { Ejemplos contextualizados } \\
\text { Velocidad del habla }\end{array}$ \\
\cline { 2 - 3 } & Estrategias institucionales & Orientación cultural
\end{tabular}

Fuente: Elaboración propia

Respecto a la subcategoría estrategias docentes, las y los docentes participantes expresaron el intento por usar un lenguaje neutro y académico, evitando el uso de modismos y regulando la velocidad del habla. Asimismo, mencionan el conocimiento respecto de las culturas de origen de sus estudiantes para así poder adaptar los ejemplos y contenidos a contextos más amplios:

Tiene que ver con esta adaptación de las explicaciones a contextos más amplio que pueden ser entendidos por distintas culturas. Los localismos en términos de explicaciones hoy en día afectan, no quiero exagerar, pero son discriminatorios entiendes si yo explico todo en términos de localismos nacionales, ejemplos de Chile eso es discriminatorio. (Profesor 1, comunicación personal, 05 de diciembre de 2020).

Esto es confirmado por un estudiante quien señala que:

...a veces es como nos tenemos que parar para que se me explique cuando hablan los de ejemplos, cuando estamos hablando de una teoría y hablamos de la ejemplificación como que se me tiene que detener porque allí ahí usan ejemplos contextualizados a la cultura chilena. (Estudiante 3, comunicación personal, 12 de enero de 2020). 
En ese sentido, la importancia de contextualizar y de considerar que en la sala de clases hay un estudiante con vivencias culturales distintas a las del grupo mayoritario, no solo es visto como una estrategia pedagógica sino también de buena relación y antidiscriminatoria.

Asimismo, otro elemento importante es la asistencia específica a nivel institucional, así como el apoyo de las direcciones correspondientes a través de sus funcionarios, quienes apoyan en el momento de la inserción: "Yo creo que es el acompañamiento de los dirigentes del programa porque los trámites del programa cuando empecé a tomar el curso habia alguien que me orientaba sobre estos trámites" (Estudiante 5, comunicación personal, 11 de enero de 2020). De esta forma, aparece otra figura de relación positiva que es un administrativo que orienta en determinados procesos:

Hay personas que enseñan a convivir acá en Chile, no solo en la universidad si no cuando tu estas en una tienda, cuando estas en un bus ¿me entiende? entonces yo creo que debería de existir ese tipo de comisario, personas que son, existen solo para estudiantes de pre grado pero no existe para post grado (Estudiante 2, comunicación personal, 9 de enero de 2020).

En ese sentido, las estrategias mencionadas no solo surgen desde la necesidad académica, sino también de la necesidad de vincularse y participar del nuevo contexto social. Las figuras administrativas son relevadas en el discurso de los estudiantes por su capacidad de resolver problemas y también porque son personas que les conectan con la nueva cultura, constituyéndose así en factores protectores y promotores de la adaptación. Sin embargo, se devela el vacío que existe en postgrado, donde parece obviarse esta necesaria relación.

\section{Discusión}

Retomando el objetivo de analizar los procesos de adaptación académica de estudiantes extranjeros de postgrado de una Universidad pública del sur de Chile, lo primero que resulta interesante discutir es el rol del lenguaje en dicho proceso. Si bien la mayoría de las y los entrevistados tiene como primera lengua el idioma español, el cual es el idioma que también se habla en Chile, las variantes lingüísticas propias de cada país (Suriani, 2006), que han favorecido históricamente la comunicación entre países de Latinoamérica, sí parecen constituir un obstaculizador cuando de procesos de aprendizajes se trata.

En este sentido estaría operando el denominado duelo lingüístico (Guerrero, 2017), en el cual la adaptación a las nuevas variables dificulta la expresión de aspectos íntimos del sujeto (como el pensamiento, las emociones, la forma de preguntar, entre otros), generando una situación de doble aprendizaje. Mientras se trata de aprender los objetivos diseñados de acuerdo con los programas, también se aprenden las nuevas lenguas o nuevas variables lingüísticas. En ese sentido, el que las y los estudiantes provengan de países que hablen español no garantiza que la barrera idiomática esté superada por sí misma.

Coherente con lo anterior, los procesos de adaptación (Ramírez, Herrera y Herrera, 2007) presentes en los estudiantes (autorregulación, integración y aislamiento) parecen depender de varios factores. En ese sentido, están las propias habilidades del estudiantado para relacionarse con otros y otras, así como decodificar las intenciones de esos otros y otras, pero también las adaptaciones de aquellos y aquellas que forman parte de la cultura mayoritaria.

De acuerdo a lo anterior, la experiencia de las y los participantes del estudio describe un entorno sociocultural con poca flexibilidad, por tanto, la adaptación, en lugar de conformarse como un proceso interactivo de cambio entre individuo y su entorno (Builes, Manrique y Henao, 2017), se transforma en un proceso unidireccional que afecta al estudiantado migrante y cuya estrategia en general tiende a ser de evitación.

Es importante considerar que la/el estudiante extranjero/a inicia un proceso en el cual intenta equilibrar su herencia cultural originaria con la de la cultura dominante (Castro, 2011). De esa forma no es extraño que los intentos de integración utilicen como medio el rescate y presentación de la herencia cultural originaria, la cual de ser recibida favorablemente por los pares o docentes para permitir el proceso de integración; este 
fenómeno ya ha sido descrito en Saldivia-Muñoz y Médor (2020). En ese sentido, sería importante que pares y profesores/as tengan conocimiento respecto de este proceso.

El desinterés o el bloque de la experiencia originaria del estudiantado migrante, pueden iniciar automáticamente un proceso de adaptación autorregulado, en el cual el/la estudiante ajusta las interacciones a lo estrictamente académico (García-Martín, 2012). En un caso extremo, el/la estudiante migrante optará por aislarse, resultando este el mecanismo de adaptación elegido para sortear el proceso de estudiar en el extranjero.

Si bien en una interacción pedagógica pudiera pensarse que las y los compañeros constituyen una red de apoyo importante, las y los participantes reportan un vínculo más estrecho con sus docentes. Esto ha sido expuesto en Bustos y Gairín (2017), y en ese sentido resulta importante hacer explícito este rol en las y los docentes, puesto que su figura puede constituir un elemento a favorecer más procesos de adaptación ligados a la integración, así como a contener aspectos emocionales, al mismo tiempo que puede contribuir a reducir los procesos de adaptación orientados al aislamiento.

Resulta interesante discutir cómo los procesos de aislamiento también constituyen un proceso de adaptación, el cual tiene costos importantes para la salud mental del estudiantado. La adaptación como se ha expuesto, está relacionada con el bienestar (Castro, 2011). En ese sentido, cuando un estudiante se adapta, eligiendo o aceptando el aislamiento, lo hace con la finalidad de evitar un mal mayor para su bienestar como la humillación, el fracaso o el desprecio que puede provocar la interacción forzada. Así, en un sistema sociocultural rígido en que la adaptación es un esfuerzo individual de la persona migrante, la rigidez permite mantener la cultura intacta y asegurar que las y los nuevos integrantes se acomoden a ella, disponiendo para ello de procesos de discriminación (Bustos y Gairín, 2017).

Asumir el costo de estos procesos negativos durante una adaptación, tiene por efecto una aculturación, en el cual el estudiantado, con la finalidad de integrarse, necesita realizar un proceso de adaptación denominado aculturación (Sosa, Fernández y Zubieta, 2014). Como el estudiantado se da cuenta de que en su proceso de integración su experiencia inicial no es recibida, busca vivencias en la nueva cultura, vaciándose de su cultura original con la finalidad de integrarse. Cuando el estudiantado no está dispuesto a hacer este proceso, y el grupo dominante no responde, se cae en el aislamiento. Es decir, el estudiantado elige o acepta aislarse para evitar los problemas que supone la integración y poder continuar estudiando.

Independiente de las preferencias personales, cuando el aislamiento es producido por el contexto, las personas pueden sufrir diversos problemas de salud mental, tales como depresión, dependencia a personas, insomnio, así como problemas físicos de salud (Géne-Badia, Ruíz-Sánchez, Obiols-Masó, Oliveras y Legarda, 2016). En ese sentido, el estudiantado que usa el aislamiento como proceso adaptativo, lo hace a costa de su propia salud, lo cual tendrá un impacto en su proceso de aprendizaje.

Por tal motivo, sería deseable que las y los profesores no solo posean estrategias para adaptar sus ejemplos a un espectro regional más amplio que el propio país o de adaptación de sus variantes lingüísticas hacia un español más neutro, sino también es necesario que pongan más énfasis en su rol como red de apoyo principal de los y las estudiantes. Asimismo, las instituciones que se abren al intercambio de estudiantes necesitan explicitar este fenómeno y considerarlo en sus políticas institucionales.

Los apoyos institucionales han sido descritos como favorables para las y los estudiantes (Bustos, 2016); de esta forma, las y los profesores que tienen estudiantes migrantes en sus clases deberían tener información respecto de las culturas de origen de estos para poder realizar ajustes a sus ejemplos o explicaciones. En otros contextos, como el caso de Perú, esto se ha logrado con facilitadores interculturales (Sepúlveda y Cabieses, 2019) que son personas que conocen y son parte de ambas culturas, por tanto tienen la posibilidad de colaborar en la conexión cuando aparecen nuevos/as integrantes. Las universidades que forman parte de los programas de intercambio deben poner énfasis en el lenguaje de sus docentes para neutralizar y promover la velocidad del habla, y asimismo disponer de tiempos para el apoyo por parte de sus profesores/as hacia sus estudiantes, teniendo a estos primeros como principal red de apoyo de los segundos. 
En la Tabla 3 se sistematizan orientaciones específicas producto del análisis realizado y que pueden ser de utilidad para las instituciones y docentes.

TABLA 3

Orientaciones para instituciones y docentes

Momento Estrategia

Interiorizarse sobre la cultura de origen de las y los estudiantes (palabras en común, palabras con significados distintos, aspectos de formalidad como saludar, dirigirse a la/el otro/a, entre otros)

Antes de la clase

Contextualización de los contenidos a las culturas de origen de las y los estudiantes. Esto implica establecer alguna relación entre el conteniclo a aprender con la cultura de las y los estudiantes.

Ejemplos contextualizados. Esto implica indagar en ejemplos históricos o del momento actual vivido por el pais de origen o bien estableciendo paralelos entre cómo un mismo fenómeno se puede abordar entre uno o más paises.

Regular modismos, velocidad y modulación por parte de la/el profesor y solicitar también para los compañeros y compañeras en momentos de formalidad.

Brindar aclaraciones respecto de lo tratado en clases. Diseñar cierres en que se repitan ideas centrales y en que el cuerpo estudiantil pueda establecer dudas y certezas

Establecer momentos para la comunicación con estudiantes cuya primera lengua no sea el

Durante las clases español. Implementar tutorias, acompañadas de software de traducción para aclarar ideas o procedimientos de la clase.

Valorar las variables idiomáticas escritas y habladas de estudiantes cuya primera lengua no es el español. Motivar el uso de material pedagógico o de comunicación oral o conceptos en el idioma original de la/el estudiante cuando este no es español.

Interiorizarse en la vida personal de la/el estudiante, sus facilitadores y obstaculizadores con la finalidad de diseñar las clases tomando en consideración estos hechos.

Especial atención a la discriminación. Explicar la tolerancia cero a la discriminación en el aula y abrir canales de comunicación para expresar dichas situaciones. Asimismo, estar más atento/a a abordar cualquier situación que pudiera parecer discriminatoria.

Identificar competencias lingüisticas de las y los estudiantes. Analizar producciones orales y escritas de las y los estudiantes para identificar niveles de abstracción o concretismo lingüistico, conceptos aprendidos y no aprendidos, modismos que actúen como obstaculizadores, entre otros

Después de la clase Aclarar temáticas brindadas en clase. Disponer espacios virtuales o en horarios alternos para aclarar temáticas no resueltas.

Solicitar retroalimentación a las y los estudiantes. Incorporar la opinión de estudiantes migrantes para la mejora de las prácticas, respecto a temas como en los ejemplos brindados, el uso de modismos, ritmo del habla y de la relación pedagógica en general. Disponer de facilitadores/as interculturales. Esto es, personas que conozcan las culturas de origen del estudiantado y puedan asesorar en aspectos lingüisticos y de contexto para docentes.

Institucional Incorporar horas adicionales a los cursos en que participan estudiantes migrantes, especialmente para las tutorias. Establecer protocolos antidiscriminación y códigos de ética para regular la relación en ese aspecto.

Fuente: Elaboración propia 


\section{Conclusiones}

En el presente artículo se analizaron los procesos de adaptación académica de estudiantes extranjeros de postgrado de una universidad pública del sur de Chile. En este sentido, se puede concluir que los procesos adaptativos que experimentan estos y estas estudiantes se categorizan en una Adaptación académica, una Adaptación Sociocultural y una Adaptación Psicológica.

Si se entiende la adaptación como un proceso recíproco entre el individuo y su contexto orientado a realizar modificaciones conductuales para garantizar el bienestar, se concluye que en percepción de las y los estudiantes migrantes que forman parte de esta investigación, el mayor esfuerzo adaptativo está realizado por ellas y ellos como individuos y no por los contextos. Así, el proceso adaptativo deja de ser recíproco y pasa a ser individual, lo cual podría explicar por qué dentro de las estrategias se encuentra la autorregulación como forma para limitar la interacción social a lo estrictamente académico o bien el aislamiento como una forma de evitar el fracaso social.

En ese sentido, la relación académica parece ser la más recíproca y esto se da cuando los profesores y profesoras tienen información sobre los procesos de las y los estudiantes migrantes concretándose esa información en la adaptación de ejemplos, contextualización de la información, control de los conceptos y la velocidad de habla. Si bien esto no se da con todos las y los profesores y parece estar influido fuertemente por las oportunidades de las y los docentes de haberse relacionado anteriormente en contextos interculturales, esta experiencia permite vislumbrar la diferencia entre un entorno cultural que promueve una adaptación recíproca y uno que no.

Las consecuencias de una adaptación individual y menos recíproca tienen relación con esfuerzos importantes de las y los estudiantes por racionalizar su situación de migración y de entender las conductas discriminatorias, concretándose esta racionalización en conductas de aislamiento o de focalización en lo académico. En ese sentido podría pensarse que las y los estudiantes migrantes de postgrado podrían estar más expuestos/as a problemas de salud mental, puesto que adicional al esfuerzo que deben poner en su proceso de estudio y en la lejanía de su lugar de origen, deben sumar la racionalización de experiencias discriminatorias provenientes principalmente del entorno sociocultural. Desde ahí que el contexto académico, especialmente de parte del cuerpo docente, puede constituir un factor protector.

Las principales recomendaciones van dirigidas a acciones institucionales de protección y orientación de la/el estudiante migrante. Se hace necesario que las instituciones universitarias pongan especial atención en sus procesos de acompañamiento y acogida de estudiantes migrantes, lo cual tiene implicaciones en el fortalecimiento de las clases con preparación de los profesores y profesoras para acercar la temática de aprendizaje a la cultura de todos los y las estudiantes en el aula, incorporar recursos para tutorías que permitan un vínculo más cercano, así como la incorporación de facilitadores/as interculturales.

Los desafíos socioculturales son menos abordables por las instituciones, puesto que tienen relación con la vida al exterior de la universidad. Sin embargo, sí es posible que a través del facilitador intercultural se puedan disponer apoyos para comprender la idiosincrasia, intenciones, formas de integración y, asimismo, mecanismos de denuncia y de acompañamiento cuando se vivencien experiencias de discriminación.

La investigación disponible sobre adaptación de estudiantes migrantes se focaliza bastante en el componente afectivo y en las relaciones con pares. Esta investigación ha contribuido con nuevo conocimiento para reforzar esta idea, y ha aportado en la relación de ese componente afectivo con el aprendizaje. Así, la integración de las y los estudiantes migrantes no es una cuestión únicamente afectiva y dependiente de las habilidades propias de cada estudiante, sino que además es parte de un entramado de gestión que parte desde las políticas institucionales hacia las decisiones pedagógicas en el aula y el rol del y la docente. 


\section{REFERENCIAS BIBLIOGRÁFICAS}

Aldunate, R., Contreras, G., de la Huerta, C. y Tapia, M. (2018). Caracterización de la migración reciente en Chile. Economia Chilena. 21(3). 110-123. Recuperado de https://www.bcentral.cl/c/document_library/get_file?uuid $=\mathrm{d} 08 \mathrm{f} 926 \mathrm{f}-46 \mathrm{ea}-2461-\mathrm{cc} 18-60 \mathrm{ad} 644 \mathrm{~b} 2677$ \&groupId $=33528$

Aninat, I. y Vergara, R. (2019). Inmigración en Chile. Una mirada multidimensional. Chile: CFE.

Arenas, P. y Urzúa M. (2016). Estrategias de aculturación e identidad étnica: un estudio en migrantes sur-sur en el norte de Chile. Universitas Psychologica., 15(1), 15-25. Recuperado de http://www.scielo.org.co/pdf/rups/v15 $\mathrm{n} 1 / \mathrm{v} 15 \mathrm{n} 1 \mathrm{a} 09 . \mathrm{pdf}$

Banco Mundial. (2017). Chile, Panorama General. Recuperado de https://www.bancomundial.org/es/country/chil e/overview

Banks, M. (2010). Los datos visuales en la investigación Cualitativa. Madrid: Morata

Builes, I., Manríquez, H. y Henao, C.M. (2017). Individuación y adaptación: Entre determinaciones y contingencias. Nómadas. Revista Cientifica de Ciencias Sociales y Jurídicas. 51(2). 1-23. Recuperado de https://www.redalyc.o $\mathrm{rg} / \mathrm{pdf} / 181 / 18153284002 . \mathrm{pdf}$

Bustos, R. (2016). Motivación escolar y rendimiento: impacto de metas académicas, de estrategias de aprendizaje y autoeficacia (Tesis de Doctorado). Universidad de Coruña. Recuperado de http://hdl.handle.net/2183/16884

Bustos, R. y Gairín, J. (2017). Adaptación académica de estudiantes migrantes en contexto de frontera. Calidad en la educación, 46, 193-220. Recuperado de https://scielo.conicyt.cl/pdf/caledu/n46/0718-4565-caledu-46-001 93.pdf

Castillo, D., Santa-Cruz, E. y Vega, A. (2018). Estudiantes Migrantes en las Escuelas Públicas Chilenas. Calidad en la Educación, 49, 18-49. Recuperado de https://scielo.conicyt.cl/pdf/caledu/n49/0718-4565-caledu-49-00018 .pdf

Castro, S. (2011). Estrategias de aculturación y adaptación psicológica y Sociocultural de estudiantes extranjeros en la Argentina. Interdisciplinaria, 28(1). 115-130. Recuperado de https://www.redalyc.org/articulo.oa?Id=180/ 18022327007

Castro, A. y Lupano, L. (2013). Predictores de Adaptación de Estudiantes Extranjeros.Interdisciplinaria,30(2). 265-281. Recuperado de https://www.redalyc.org/pdf/180/18029870006.pdf

Comisión Económica para América Latina [CEPAL] (2018). La agenda 2030 y los Objetivos del desarrollo sostenible. Una oportunidad para latinoamérica y el Caribe. Recuperado de https://repositorio.cepal.org/bitstream/handl e/11362/40155/24/S1801141_es.pdf

Consejo Nacional de Educación [CNED] (2019). Resumen Matricula de Pregrado de Educación Superior. Recuperado de https://www.cned.cl/sites/default/files/principales_tendencias_de_matricula_es_2019.pdf

Escobar, J. y Cuervo, E. (2008). Validez de Contenido y Juicio de Expertos: Una Aproximación A Su Utilización. Avances en Medición, 6, 27-36. Recuperado de https://dialnet.unirioja.es/servlet/articulo?codigo $=2981181$

Espinoza, O. y González, L.E. (2016). La educación superior en chile y la compleja transición desde el régimen de autofinanciamiento hacia el régimen de gratuidad. Revista Latinoamericana de Educación Comparada, 7(10), 35-51. Recuperado de https://dialnet.unirioja.es/descarga/articulo/6562408.pdf

Ferrer, R., Palacios, J., Hoyos, O. y Madariaga, C. (2014). Procesos de aculturación y adaptación del inmigrante: Características individuales y redes sociales. Psicología desde el Caribe, 31(3), 557-576. Recuperado de http://d x.doi.org/10.14482/psdc.31.3.4766

García-Martín, M. (2012). La Autorregulación Académica Como Variable Explicativa de los Procesos de Aprendizaje Universitario. Profesorado. Revista de Currículum y Formación de Profesorado, 16(1), 203-221. Recuperado de : https://www.redalyc.org/articulo.oa?id=567/5672437

Géne-Badia, J., Ruíz-Sánchez, M., Obiols-Masó, N., Oliveras, L. y Legarda, E. (2016). Aislamiento social y soledad ¿qué podemos hacer los equipos de atención primaria?. Atención primaria, 48(9), 604-609. Recuperado de https://www.elsevier.es/es-revista-atencion-primaria-27-articulo-aislamiento-social-soledad-qu e-podemos-S0212656716301809 
Guerrero, C. (2017). Inmigrantes en una Escuela Multicultural en Santiago de Chile: Estudio Antropológico-Lingüistico de Representaciones Sociales En Torno A Variedades Lingüisticas en Contacto/Conflicto. (Tesis de licenciatura) Universidad de Chile. Recuperado de http://repositorio.uchile.cl/handle/2250/169912

Instituto Nacional de Estadísticas [INE] (2019). Informe Metodológico de Estimación de Personas Extranjeras Residentes en Chile al 31 De Diciembre 2018. Recuperado de https://www.extranjeria.gob.cl/media/2019/07/E stimaci\%C3\%B3n-Poblaci\%C3\%B3n-Extranjera-en-Chile.pdf

Kulshrestha, S. (1979). Educational Psychology. Meerut: Loyal Book Depot

Jiménez, M. I. y López-Zafra, E. (2011). Actitudes sociales y adaptación social en adolescentes españoles: El papel de la inteligencia emocional percibida. Revista de Psicología Social, 26(1), 105-117. doi: https://doi.org/10.1174/ 021347411794078417

Joiko, S. y Vásquez, A. (2016). Acceso y elección escolar de familias migrantes en Chile: "No tuve problemas porque la escuela es abierta, porque acepta muchas nacionalidades". Calidad en Educación, 45, 132-137. Recuperado de http://dx.doi.org/10.4067/S0718-45652016000200005

Ley N 20.050. Diario Oficial de la República de Chile, Santiago, Chile, 26 de agosto de 2015.

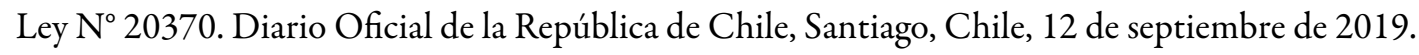

Mackernan, J. (1989). "El estudio de casos" en Investigación - acción y currículum. Madrid: Morata.

Mulholland, E. y Hernández-Julian, R. (2013). Does economic freedom lead to selective migration by education? Journal of regional analysis \& policy, 43(1), 65-87. https://doi.org/10.22004/ag.econ.243949

Ministerio de Educación [MINEDUC] (2018). Politica nacional de estudiantes extranjeros 2018-2022. Recuperado de https://bit.ly/3bJ7G5G

Mondaca, C., Muñoz, W., Gajardo, Y. y Gairín, J. (2018). Estrategias y prácticas de inclusión y estudiantes migrantes en las escuelas de Arica y Parinacota, frontera norte de Chile. Estudios Atacameños, 57, 181-201. Recuperado de http://dx.doi.org/10.4067/S0718-10432018005000101

Monje, C.A. (2011). Metodología de la Investigación Cuantitativa y Cualitativa. Madrid: Neiva.

Nada, C.I. y Araujo, H.C. (2018). Migration and education: a narrative approach to the experience of foreign students in Portugal. London Review of Education, 16(2), 308-324. Recuperado de https://doi.org/10.18546/LRE.16.2 .10

Niño,R. (2011). Metodología de la Investigación: Diseño y Ejecución. Bogotá: Ediciones de la U.

Organización para la Cooperación y el Desarrollo Económico [OCDE] (2017). Revisión de los recursos escolares: Chile. Recuperado de https://bit.ly/3tikAgX

Organización Internacional para las Migraciones [OIM] (2018). Informe de las Migraciones en el mundo 2018. Recuperado de https://publications.iom.int/system/files/pdf/wmr_2018_sp.pdf

Organización Internacional para las Migraciones [OIM] (2019). Informe sobre las Migraciones en el mundo 2020. Recuperado de https://publications.iom.int/system/files/pdf/wmr_2020_es.pdf

Organización de las Naciones Unidas [ONU] (1948). Declaración Universal de los Derechos Humanos. Recuperado de https://www.ohchr.org/EN/UDHR/Documents/UDHR_Translations/spn.pdf

Organización de las Naciones Unidas [ONU] (2016). Declaración de Nueva York para los refugiados y migrantes. Recuperado de https://www.acnur.org/5b4d0eee4.pdf

Organización de las Naciones Unidas [ONU] (2017). Fortalecimiento de la cooperación internacional y la gobernanza de la migración con miras a la adopción de un pacto mundial para una migración segura, ordenada y regular en 2018. Recuperado de https://refugeesmigrants.un.org/sites/default/files/iom_idm_2017_spanish_14nov17.p $\mathrm{df}$

Organización de las Naciones Unidas para la Educación, Ciencia y la Cultura [UNESCO] (2018). Red Regional de Educación para la Ciudadanía Mundial para la América Latina y el Caribe "Hacia un mundo sin muros: educación para la ciudadanía mundial en el ODS 4 - Agenda E2030” Recuperado de https://bit.ly/3rVgZ8F 
Organización de las Naciones Unidas para la Educación, Ciencia y la Cultura [UNESCO] (2019). Informe de seguimiento de la educación en el mundo, 2019: Migración, desplazamientos y educación: construyendo puentes, no muros. Recuperado de https://es.unesco.org/gem-report/

Poblete, R. (2018). El trabajo con la diversidad desde el currículo en escuelas con presencia de niños y niñas migrantes: estudio de casos en escuelas de Santiago de Chile. Perfiles Educativos, 40(159), 51-65. Recuperado de http://ww w.scielo.org.mx/scielo.php?.pid=S0185-26982018000100051\&script $=$ sci_arttext

Ramírez, M.I., Herrera, F. y Herrera. I. (2007). ¿Qué ocurre con la adaptación y el rendimiento académico de los alumnos, en un contexto educativo pluricultural? Revista Iberoamericana de educación, 33(2), 1-17. Recuperado de https://rieoei.org/RIE/article/view/2994/3898

Ruíz, J. (2007). Metodología de la Investigación Cualitativa. Bilbao: Universidad de Deusto

Saldivia-Muñoz, F.H. y Médor, P.R. (2020). Proceso de inclusión de estudiantes Haitianos en establecimientos municipales pertenecientes a enseñanza media de la XIV región de los Ríos, con énfasis en educación física. Infancia, Educación y Aprendizaje, 7(1), 132-159. Recuperado de https://doi.org/10.22370/ieya.2021.7.1.1995

Salazar-Salas, G. (2015). Nicaragüenses inmigrantes en Costa Rica: Patrones de participación en actividades recreativas. Revista Educación, 39(1), 91-119. doi: http://dx.doi.org/10.15517/revedu.v39i1.17850

Sepúlveda, C. y Cabieses, B. (2019). Rol del facilitador intercultural para migrantes internacionales en centros de salud chilenos: perspectivas de cuatro grupos de actores clave. Revista Peruana de Medicina Experimentaly Salud Pública, 36(4). Recuperado de https://doi.org/10.17843/rpmesp.2019.364.4683

Stefoni, C. y Corvalán, J. (2019). Estado del Arte sobre inserción de niños y niñas migrantes en el sistema escolar chileno. Estudios Pedagógicos, 45(3), 201-215. Recuperado de http://dx.doi.org/10.4067/S0718-07052019000 300201

Sosa, F., Fernández, O. y Zubieta, E. (2014). Bienestar social y aculturación psicológica en estudiantes universitarios migrantes. Liberabit, 20(1), 151-163. Recuperado de https://bit.ly/3vkcI0A

Suriani, B. (2006). El tratamiento de la variación lingüística en intercambios sociales. Fundamentos en Humanidades, 9(1), 27-41. Recuperado de https://dialnet.unirioja.es/servlet/autor?codigo=1897677

Tinto, A. (2012). El análisis de contenido como herramienta de utilidad para la realización de una investigación descriptiva. Un ejemplo de aplicación práctica utilizado para conocer las investigaciones realizadas sobre la imagen de marca de España y el efecto país de origen. Provincia, (29), 135-173. Recuperado de http://www.re dalyc.org/pdf/555/55530465007.pdf

Torres, M. (2012). La migración y sus efectos en la cultura. Sociológica, 27(77), 301-306. Recuperado de https://bit .ly/2OLFg2e

Vásquez-De Kartzow, R., Castillo-Duran, C. y Lera, L. (2015). Migraciones de países de América Latina. Características de la población pediátrica. Revista Chilena de Pediatría, 86(5), 325-330. Recuperado de http:/ /dx.doi.org/10.1016/j.rchipe.2015.07.007

Williamson, G. (2019). Formación Ciudadana en Comunidades Educativas: Expresiones y reflexiones desde la Araucania. Temuco: LOM.

\section{INFORMACIÓN ADICIONAL}

Cómo citar: Guevara Ortiz, O.I. y Alfaro Urrutia, J.E. (2021). Análisis de los procesos adaptativos en estudiantes migrantes de postgrado de una Universidad pública del sur de Chile. Revista Educación, 45(2). Recuperado de http://doi.org/10.15517/revedu.v45i1.43617 